\title{
VOLUME ESTIMATION BY WAVELET TRANSFORM OF DOPPLER HEART SOUND DURING VENOUS AIR EMBOLISM IN DOGS
}

\author{
Francis H. Y. Chan ${ }^{1}$, Brent C. B. Chan ${ }^{2}$, P. W. Lui ${ }^{3}$, Paul W. F. Poon ${ }^{4}$, F. K. Lam ${ }^{1}$ \\ ${ }^{1}$ Dept. of Electrical \& Electronic Engineering, The University of Hong Kong, Pokfulam Rd., Hong Kong \\ ${ }^{2}$ Dept. of Computer Science, City University of Hong Kong, Tat Chee Avenue, Kowloon, Hong Kong \\ ${ }^{3}$ Dept. of Anesthesiology, Veterans General Hospital-Taipei, 201 Shih-pai Rd. Sec. 2, Taipei, Taiwan \\ ${ }^{4}$ Dept. of Physiology, National Cheng Kung University, 1 University Rd., Tainan, Taiwan \\ ${ }^{1}$ E-mail: fhychan@eee.hku.hk
}

\begin{abstract}
The Doppler heart sound signals detected by the precordial Doppler ultrasound method under simulated sub-clinical and clinically significant venous air embolism were studied in anesthetized dogs. Signal processing using wavelet transform enhanced the contrast of embolic to normal signal, facilitating automatic detection and extraction of embolic signal simply by thresholding. Linear relationship of good correlation coefficient was obtained in $\log -\log$ scale between the subclinical volume of injected air and the corresponding embolic signal power in all dogs. The calibration curve was found to be good estimate of the volume of embolic air during simulated clinically significant venous air embolism. Hence, we overcame the need of constant human attention for detecting venous air embolism and the lack of quantitative information on the volume of embolic air in the traditional precordial Doppler ultrasound method by the present approach.
\end{abstract}

Keywords - air embolism, wavelet transform, precordial Doppler ultrasound, Doppler heart sound.

\section{INTRODUCTION}

Venous air embolism (VAE) is hazardous when occurring in large quantity during surgery. It can occur through an opened vein with pressure being lower than ambient, causing air to be sucked into the circulatory system. It causes injury primarily by obstruction of blood flow from the right side of the heart to the left, resulting in considerable hypoxemia from mismatched ventilation-perfusion. With large amount of emboli, systemic hypotension, myocardial ischemia, and arrhythmia occur and can result in death.

Precordial Doppler ultrasound (PDU) is one of the most sensitive and non-invasive methods for detecting VAE [1-3], but it requires constant attention of anesthesiologists listening to subtle changes in the Doppler heart sound (DHS). Besides, it cannot provide the volume of air entering the heart chamber. Such information is crucial as anesthesiologists usually act only when clinically significant embolic air volume is present [4].

Common clinical research studies generally focused on the sensitivity of PDU method towards VAE [1-3, 5-7] while only very limited works were devoted to DHS signal processing for VAE detection. Pattern recognition algorithms $[8,9]$ were applied to DHS signals for detecting VAE, which allowed unattended operation with sensitivity comparable to human observers. However, estimation of embolic air volume seems to be a difficult task as hardly any detailed research work on this aspect can be found.

In view of the above situation, we developed a fast detection algorithm with good sensitivity (down to $0.01 \mathrm{ml}$ of air) using wavelet transform of DHS [10]. With further development and modification of this algorithm [11], we managed to implement it as a real-time monitor for detecting VAE [12]. Using this monitor, we studied the performance of VAE detection during sub-clinical bolus injection and clinically significant continuous infusion of air in dogs [13]. In the present study, we actually estimated the volume of embolic air during several simulated periods of clinically significant VAE with different air dosage based on the calibration curves derived from prior injections of subclinically small air volumes. We also demonstrated that the air dosage of prior sub-clinical injections can be reduced to as low as $0.20 \mathrm{ml}(0.80 \mathrm{ml}$ in our previous studies $)$ in order to minimize the adverse effect (if any) on the dogs.

\section{MATERIALS AND METHODS}

The study was approved by the Animal Research Committee of the Veterans General Hospital-Taipei. Three mongrel dogs weighing $11.67 \pm 2.08 \mathrm{~kg}$ were anesthetized with pentobarbital $(40 \mathrm{mg} / \mathrm{kg}$, i.v. $)$ and placed in the supine position for the experiment. The trachea was intubated with a cuffed tracheal tube using vecuronium $(0.1 \mathrm{mg} / \mathrm{kg}$, i.v. $)$ and the lungs were ventilated by a mechanical ventilator (Model 34RH, Harvard Apparatus, Natick, MA). The tidal volume was set at 15 to $20 \mathrm{ml} / \mathrm{kg}$. Rectal temperature was controlled at $37 \pm 1{ }^{\circ} \mathrm{C}$ using a heating lamp and $0.9 \%$ normal saline in $5 \%$ glucose solution was infused $\left(3 \mathrm{ml} \mathrm{kg}^{-1} \mathrm{~h}^{-1}\right.$, i.v.) to maintain a normal body fluid status. Cardiac rhythm was monitored by lead II electrocardiograph (Model 90601A, SpaceLab, Redmond, WA).

A 16-gauge Teflon catheter (Arrow International, Reading, PA) was inserted via the right external jugular vein for air 
infusion. Generous quantity of coupling gel (Aquasonic 100, Parker Lab, Orange, NJ) was applied thoroughly to the circular face of the PDU probe (Model P81, $2.4 \mathrm{MHz}$, VERSATONE $^{\otimes}$ Doppler, MedaSonics, Fremont, CA), which was then pressed against the shaved skin at the right lateral border of the sternum. The correct placement of the probe over the heart was confirmed by a clearly audible sound of turbulence induced by rapid injection of $10-\mathrm{ml}$ normal saline into the external jugular vein via the catheter. Micropore tapes were used to secure the probe over the chest. The line output of the PDU machine was connected to a tape recorder (WM-D6C, Sony Corp., Japan) for recording the DHS during the entire experiment. The overall frequency response of the recording system was flat $( \pm 3 \mathrm{~dB})$ from $20 \mathrm{~Hz}$ to $18 \mathrm{kHz}$.

After obtaining a 5-min control baseline period of DHS, a series of sub-clinically small boluses of air $(0.01,0.015,0.02$, $0.03,0.04,0.05,0.07,0.10,0.15,0.20 \mathrm{ml}$ ) were injected into the external jugular vein through the catheter. Each injection was made by means of a microsyringe (Hamilton, Reno, NV) at 1-min interval. In order to increase the reliability and accuracy, injection for each dosage was repeated four times. After a 10-min stabilization period, a series of large air volume $(2.00,3.20,4.80 \mathrm{ml})$ were infused gradually, each lasting for $5 \mathrm{~min}$, so as to simulate a series of clinically significant VAE of different volumes. Intermissions of $3 \mathrm{~min}$ were allowed between successive infusions. The 5-min interval for each infusion was divided into twenty $15-\mathrm{sec}$ intervals and appropriate dosage $(0.10,0.16,0.24 \mathrm{ml})$ was injected gradually over these 15 -sec intervals. For example, injecting $0.16 \mathrm{ml}$ of air gradually over $15-\mathrm{sec}$ interval for twenty times would give a simulation period of $3.20 \mathrm{ml}$ of air infused over $5 \mathrm{~min}$. The DHS signals produced by this infusion protocol corresponded closely to those encountered during actual clinical VAE, as confirmed by an experienced anesthesiologist (PWL).

The DHS signals recorded were then fed to our real-time monitor for analysis and processing [12, 13]. Briefly, these signals were firstly digitized at $4 \mathrm{kHz}, 16-$ bit resolution. Then, they were processed using the Daubechies 20-coefficient wavelets [14], which in effect suppressed the signal power of the normal DHS and enhanced those of the embolic DHS at successive scales of the wavelet transform. For each subject, the embolic DHS signal powers (embolic power in short) due to each sub-clinical bolus air injection were evaluated (repeated 4 times), and their mean (arithmetic mean (AM) and geometric mean (GlM)) were then computed. Linear regression and correlation analyses were performed on the volume of injected air and the corresponding mean embolic power. Finally, the series of embolic power data accumulated for each simulated period of clinically significant VAE in each subject were fitted to the corresponding linear trends (calibration curves) for volume estimation.

\section{RESULTS}

A representative tracing of the original and waveletprocessed DHS signals is shown in Fig. 1. Although the approximations (A1 and A2) grossly resembled the original DHS signals, the details (D1 and D2) showed striking features distinguishing embolic DHS from the normal. Essentially, the contrast of embolic to normal DHS signal power became greatly enhanced, facilitating detection and extraction of embolic power by simple thresholding. The quality of this differentiation for D1 easily exceeded that of D2, but the latter was also useful for confirming the detection made by the former. Fig. 2 shows the embolic power variation of the original DHS signals, D1, and D2 in Fig. 1. The zero embolic power in the original DHS signals implied that embolic DHS cannot be detected without the present wavelet transform method.

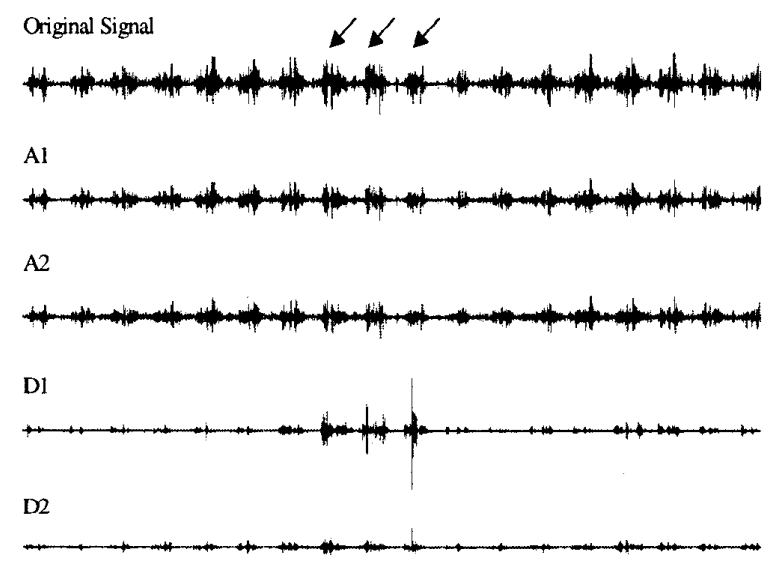

Fig. 1. The approximations (A1 and A2) and details (D1 and D2) of the DHS signals at scale $=1$ and scale $=2$. Arrows mark the embolic DHS signals after a bolus air injection of $0.02 \mathrm{ml}$.

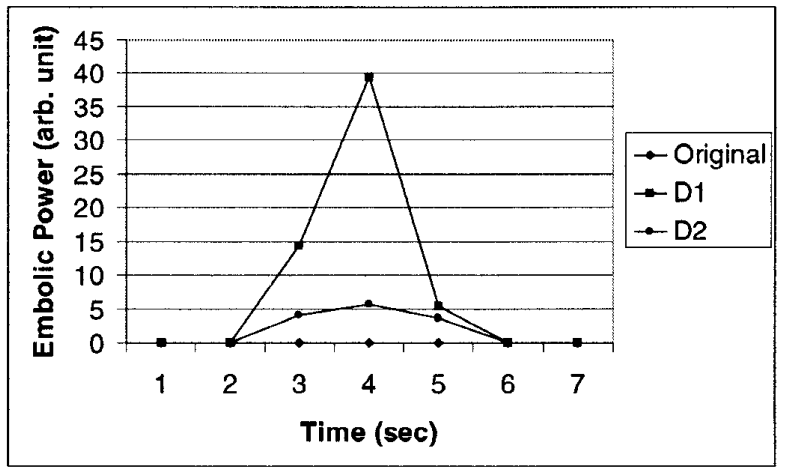

Fig. 2. Plot of the embolic power variation of the original DHS signals, D1, and D2 in Fig. 1. 
For each subject, linear relationship of good correlation coefficient was obtained in log-log scale (natural $\log$ ) between the sub-clinical volume of injected air and the corresponding embolic power computed from the D1 signals. The resulting GM calibration curves of the three subjects in this study are plotted in Fig. 3, and the linear regression and correlation results of the AM and GM cases are listed in Table 1. The small relative error (i.e., standard deviation divided by mean) of the slope and intercept, and the high correlation coefficient suggested the small inter-animal variation and the reliability of the present wavelet transform method, respectively.

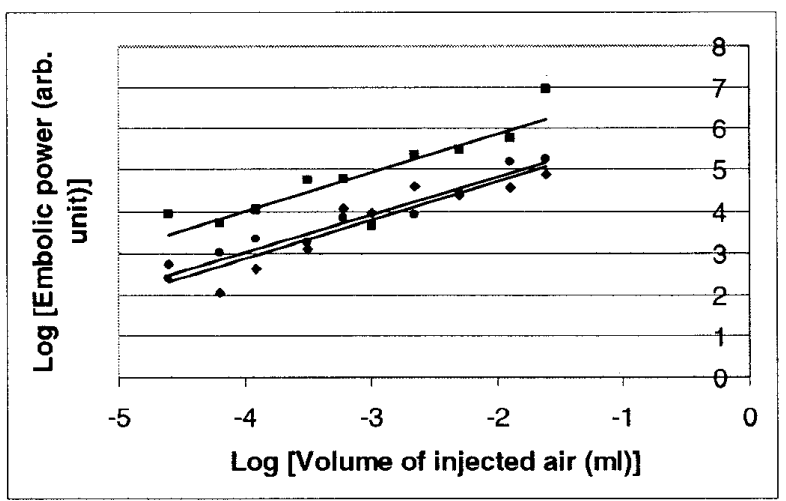

Fig. 3. Log-log plot (natural $\log$ ) of the GM embolic power against the volume of air during sub-clinical bolus injection $(0.01$ to $0.20 \mathrm{ml})$ for the three dogs. Markers $\bullet$ : Dog 1, $\mathbf{\square}$ : Dog 2, : Dog 3.

\begin{tabular}{lcccccc}
\hline & \multicolumn{2}{c}{ Slope } & \multicolumn{2}{c}{ Intercept } & \multicolumn{2}{c}{ r } \\
& AM & GM & AM & GM & AM & GM \\
\hline Dog 1 & 0.94 & 0.92 & 6.73 & 6.54 & 0.88 & 0.92 \\
Dog 2 & 0.90 & 0.93 & 7.83 & 7.70 & 0.83 & 0.86 \\
Dog 3 & 0.81 & 0.90 & 6.44 & 6.60 & 0.96 & 0.97 \\
\hline SD & 0.07 & 0.02 & 0.73 & 0.65 & & \\
Mean & 0.88 & 0.92 & 7.00 & 6.95 & & \\
RE & 0.08 & 0.02 & 0.10 & 0.09 & & \\
\hline
\end{tabular}

Table 1. Linear regression results for the $A M$ and $G M$ calibration curves in Fig. 3. Abbreviation: $\mathrm{SD}=$ standard deviation; $\mathrm{RE}=$ relative error; $r=$ correlation coefficient.

The results of volume estimation using the AM calibration curves for the simulated clinically significant VAE periods of the three dogs are shown in Fig. 4, and the numerical results of the AM and GM cases are listed in Table 2. In general, we obtained reasonable estimation for all the cases, and particularly good estimation for the $2.00 \mathrm{ml}$ cases. The estimation results due to the AM and GM calibration curves were comparable.

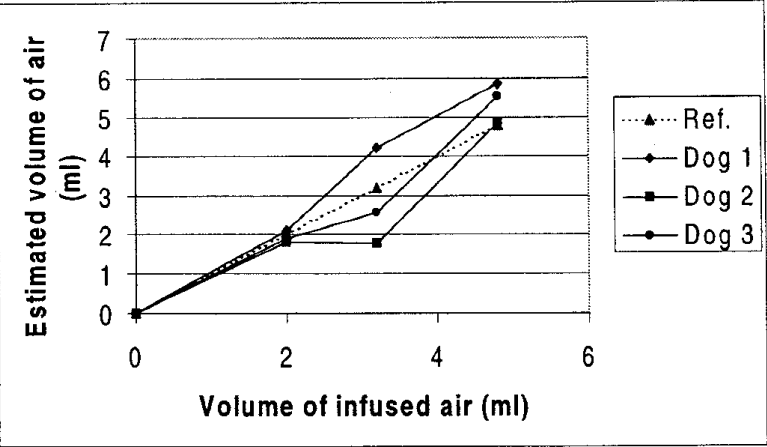

Fig. 4. Plot of the volume estimation results for the simulated clinically significant $\mathrm{VAE}$ of the three dogs using the corresponding AM calibration curves. A dotted reference line (A) showing the ideal case of volume estimation is included for comparison.

\begin{tabular}{|c|c|c|c|c|}
\hline \multicolumn{2}{|c|}{ Vol. of infused air (ml) } & $\overline{2.0}$ & 3.2 & $\overline{4.8}$ \\
\hline \multicolumn{5}{|c|}{ Estimated vol. of air (ml) } \\
\hline \multirow[t]{2}{*}{$\operatorname{Dog} 1$} & $\overline{A M}$ & 2.1 & 4.2 & 5.8 \\
\hline & GM & 2.3 & 4.5 & 6.2 \\
\hline \multirow[t]{2}{*}{$\operatorname{Dog} 2$} & AM & 1.8 & 1.8 & 4.9 \\
\hline & GM & 2.1 & 2.1 & 5.7 \\
\hline \multirow[t]{2}{*}{ Dog 3} & AM & 1.9 & 2.6 & 5.5 \\
\hline & GM & 2.0 & 2.6 & 5.1 \\
\hline \multicolumn{5}{|c|}{ Relative error } \\
\hline \multirow[t]{2}{*}{$\operatorname{Dog} 1$} & $\overline{A M}$ & 0.04 & 0.32 & 0.22 \\
\hline & GM & 0.13 & 0.41 & 0.29 \\
\hline \multirow[t]{2}{*}{ Dog 2} & AM & -0.10 & -0.45 & 0.01 \\
\hline & GM & 0.07 & -0.33 & 0.20 \\
\hline \multirow[t]{2}{*}{ Dog 3} & $\mathbf{A M}$ & -0.06 & -0.20 & 0.16 \\
\hline & GM & -0.02 & -0.20 & 0.07 \\
\hline
\end{tabular}

Table 2. Numerical results of the volume estimation and the corresponding relative error of estimation using the AM and GM calibration curves for the three dogs.

\section{DISCUSSION AND CONCLUSION}

As the linear relationship between the embolic power and the volume of injected air is in log-log scale, the averaging of the repeated data samples at each air dosage may seem to be more appropriate to use not only the traditional arithmetic mean, but also the geometric mean. In fact, both of them provide comparable volume estimation results while the latter gives better correlation coefficient and lower relative error with the three subjects in this study, as evident in Table 1.

Previously, our largest volume of sub-clinical bolus air injection was $0.80 \mathrm{ml}$ in order to study the feasibility of using these bolus calibration curves for estimating clinically significant volume of embolic air $[12,13]$. In the present 
study, we limit this largest bolus dosage to as low as $0.20 \mathrm{ml}$ while we still achieve reasonable volume estimation results. In reality, this largest bolus dosage should be brought down to as low as possible so as to minimize the adverse effect (if any) on the subjects.

The volume estimation results of the $2.00-\mathrm{ml}$ case for all the three subjects in this study are particularly outstanding, while there are some irregularities in the 3.20 and $4.80-\mathrm{ml}$ cases. For each subject, the $2.00-\mathrm{ml}$ case is the first case in the series of simulated clinically significant VAE, which is followed by the 3.20 and $4.80-\mathrm{ml}$ cases. The former case is thus performed without prior contamination of clinically significant VAE. The outstanding volume estimation performance of this case implies that our method of estimating clinically significant embolic air volume using calibration curves derived form prior sub-clinical bolus air injection is basically valid. The irregularities of the latter cases may probably be due to two main reasons. Some portion of the infused air may invariably be split into so tiny bubbles that cannot be detected by the PDU system, resulting in under-estimation. Re-circulation of air bubbles within the heart chamber as well as after the systemic circulation may contribute to over-estimation of embolic air volume. Larger volume of infused air nnay also provide higher possibility of embolic air re-circulation. In these aspects, there is a need to construct a mathematical model for the above effects in order to remedy the observed irregularities.

In conclusion, the wavelet transform of the DHS signals greatly enhanced the contrast of embolic to normal DHS signal power, facilitating automatic detection and extraction of embolic DHS simply by thresholding. Besides, the linear relationship obtained between the sub-clinical volume of injected air and the corresponding embolic power for a subject was shown to be capable of estimating clinically significant volume of embolic air for the same subject. Essentially, the two main shortcomings of the PDU method for diagnosing VAE, ramely, the need of constant human attention for VAE detection and the lack of quantitative information on the volume of embolic air, can be successfully overcome by the present: wavelet transform method.

\section{ACKNOWLEDGMENT}

This work was supported in part by National Science Council, Taiwan.

\section{REFERENCES}

[1] J. D. Michenfelder, R. H. Miller and G. A. Gronert, "Evaluation of an ultrasonic device (Doppler) for the diagnosis of venous air embolism," Anesthesiology, vol. 36, pp. 164-167, 1972.

[2] R. W. Buckland and I. M. Manners, "Venous air embolism during neurosurgery: a comparison of various methods of detection in man," Anaesthesia, vol. 31, pp. 633-643, 1976.

[3] J. B. English, D. Westenskow, M. R. Hodges and T. H. Stanley, "Comparison of venous air embolism monitoring methods in supine dogs," Anesthesiology, vol. 48, pp. 425-429, 1978

[4] R. C. Shupak, "Air embolism and its influence on anesthetic management," Current Reviews in Clinical Anesthesia, vol. 11, pp. 113-124, 1991.

[5] J. L. Chang, M. S. Albin, L. Bunegin, and T. K. Hung, "Analysis and comparison of venous air embolism detection methods," Neurosurgery, vol. 7, pp. 135-141, 1980.

[6] P. L. Gildenberg, R. P. O'Brien, W. J. Britt, and E. A. Frost, "The efficacy of Doppler monitoring for the detection of venous air embolism," Journal of Neurosurgery, vol. 54, pp. 75-78, 1981.

[7] D. A. Muzzi, T. J. Losasso, S. Black, and R. Nishimura, "Comparison of a transesophageal and precordial ultrasonic Doppler sensor in the detection of venous air embolism," Anesthesia and Analgesia, vol. 70, pp. 103 104, 1990.

[8] G. L. Gibby, and G. A. Ghani, "Computer-assisted Doppler monitoring to enhance detection of air emboli," Journal of Clinical Monitoring, vol. 4, pp. 64-73, 1988.

[9] G. L. Gibby, "Real-time automated computerized detection of venous air emboli in dogs," Journal of Clinical Monitoring, vol. 9, pp. 354-363, 1993.

[10] B. C. B. Chan, F. H. Y. Chan, F. K. Lam, P. W. Lui, and P. W. F. Poon, "Fast detection of venous air embolism in Doppler heart sound using the wavelet transform," IEEE Transactions on Biomedical Engineering, vol. 44, pp. 237-246, 1997.

[11] F. H. Y. Chan, B. C. B. Chan, F. K. Lam, P. W. F. Poon, P. W. Lui, and H. Wang, "Detection and quantification of venous air embolism by wavelet analysis of Doppler heart sound," Proceedings of $18^{\text {th }}$ Annual International Conference of the IEEE Engineering in Medicine and Biology Society, no. 95, 1996.

[12] B. C. B. Chan, F. H. Y. Chan, P. W. Lui, P. W. F. Poon, F. K. Lam, and H. Wang, "A real-time monitor using wavelet analysis of the Doppler heart sound for the detection of venous air embolism," Proceedings of the 1997 International Symposium on Circuits and Systems, pp. 189-192, 1997.

[13] P. W. Lui, B. C. B. Chan, F. H. Y. Chan, P. W. F. Poon, and F. K. Lam, "Wavelet analysis of embolic heart sound detected by precordial Doppler ultrasound during continuous venous air embolism in dogs," Anesthesia and Analgesia, vol. 86, pp. 325-331, 1998.

[14] I. Daubechies, Ten lectures on wavelets. Philadelphia, PA: SIAM, 1992. 